\title{
Evolución del peso en pacientes durante el primer año del trasplante renal
}

\author{
Pilar López Garrigós, Isabel Rojas Guadamilla, Azucena González Calero, Carmina Mas Agrafojo, Angélica \\ Gálvez Velasco, Pedro Vargas Ramírez, Ma Jesús Martínez Velasco, Gema Briceño Sánchez.
}

Enfermeras/os Servicio de Nefrología. Complejo Hospitalario de Toledo. Hospital Virgen de la Salud. Toledo

\section{Resumen}

Durante el primer año de trasplante renal es frecuente una ganancia de peso, debido, entre otros a la dieta, sedentarismo, e inmunosupresores, pudiendo aumentar los factores de riesgo del síndrome metabólico.

Objetivos. Conocer la evolución del peso de nuestros pacientes durante el primer año del trasplante, y analizar la influencia con las distintas variables asociadas al paciente, sexo, edad, IMC, tratamiento inmunosupresor entre otras. Determinar la asociación entre la ganancia de peso y la aparición de factores de riesgo, como hipertensión arterial, diabetes y dislipidemia.

Material y Método. Estudio retrospectivo y analítico. Se estudiaron 100 pacientes con un año de evolución en el trasplante, del Hospital Virgen de la Salud de Toledo. Sé analizaron datos antropométricos, demográficos y clínicos. La ganancia de peso se distribuyó según a las variables del estudio.

Resultados. La ganancia de peso al año del trasplante afecto a 93 pacientes, con una media de 7,21 kg $\pm 5,87$. El $44,1 \%$ aumento su peso por encima de la media, y el $23,7 \%$ gano $\geq 10 \mathrm{~kg}$. La prevalencia de sobrepeso y obesidad antes del trasplante fue $57 \%$ y $76 \%$ después del trasplante.

Los hombres ganaron ligeramente más peso que las mujeres. Los más jóvenes $\leq 49$ años ganaron más peso, media $9,3 \mathrm{~kg}$. Mayor incidencia de diabetes, hipertensión y dislipemias en los pacientes con sobrepeso y obesidad.

Conclusiones. La mayoría de los pacientes aumentaron significativamente de peso al año del trasplante, asociándose con varios de los factores del síndrome metabólico.

- Importante la educación, por parte de la enfermería, del paciente para fomentar hábitos de vida saludable.

Correspondencia:

Pilar López Garrigós

Calle Albarracín $n^{\circ} 7$

45122. Argés. Toledo

E-mail: pilarplg@hotmail.com
PALABRAS CLAVE

- TRASPLANTE RENAL

- GANANCIA DE PESO

- FACTORES DE RIESGO

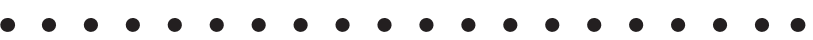

Evolution of the kidney transplant patients' weight during the first year

\section{Abstract}

During the first year of renal transplantation is frequent a weight gain, due among others to the diet, sedentary lifestyle and immunosuppressant, causing increased risk factors of metabolic syndrome.

Aim. To know the evolution of the our patients' weight during the first year after transplantation, and to analyze the influence of different variables associated with the patient, sex, age, BMI, and other immunosuppressive therapy. Determine the association between weight gain and the presence of risk factors such as hypertension, diabetes and dyslipidemia.

Material and methods. Retrospective and analytical study was performed. 100 patients were studied with a year of evolution in transplantation at the Hospital Virgen de la Salud (Toledo). Anthropometric, demographic and clinical data were analyzed. Weight gain was distributed according to the study variables.

Results. Weight gain one year after transplantation occurred in 93 patients, with a mean of $7.21 \pm 5.87$ $\mathrm{kg} .44 .1 \%$ of patients gained weight above the mean, and $23.7 \%$ gained $\geq 10 \mathrm{~kg}$. Pre-transplant prevalence of overweight and obesity was $57 \%$ and $76 \%$ posttransplant. Men gained slightly more weight than women. Younger $\leq 49$ years gained more weight, mean $9.3 \mathrm{~kg}$. Incidences of diabetes, hypertension and dyslipidemia in patients with overweight and obesity were increased.

Conclusions. Most patients gained weight significantly one year after transplantation and linked to several factors of metabolic syndrome.

Patient education performed by nurses is important to promote healthy lifestyles. 


\section{KEYWORDS}

\section{- RENAL TRANSPLANTATION \\ - WEIGHT GAIN \\ - RISK FACTORS}

\section{Introducción}

A finales del siglo XIX y principios del XX se observa el efecto nocivo del exceso de peso corporal y se relaciona a la obesidad con complicaciones de salud que aumenta la morbilidad y mortalidad del ser humano.

La OMS (Organización Mundial de la Salud) define "La obesidad y sobrepeso como una acumulación anormal 0 excesiva de grasa que puede ser perjudicial para la salud," siendo el "problema de salud con mayor incidencia" en todo el mundo. Actualmente se define la obesidad cuando el Índice de Masa Corporal es $\geq 30 \mathrm{~kg} / \mathrm{m}^{2}$, (IMC) ${ }^{1}$.

El incremento excesivo de peso corporal ha traído trastornos metabólicos que afectan a la calidad de vida de la población, asociándose a un grupo de comorbilidades: Enfermedad Cardiovascular (ECV), Síndrome Metabólico (SM), Diabetes Mellitus (DM), Enfermedad Renal Crónica ERC e Hipertensión Arterial (HTA). ${ }^{1}{ }^{2}$.La obesidad por si sola se ha convertido en un factor de riesgo independiente de desarrollo de enfermedad cardiovascular ${ }^{4}$, también se le ha asociado con una menor supervivencia del injerto y un incremento de complicaciones posquirúrgicas ${ }^{1,3}$.

En la etiología de la ganancia de peso intervienen múltiples factores, debido a que se trata de un desorden complejo en el que interaccionan principalmente factores genéticos, epidemiológicos (sexo, raza, edad) y ambientales (hábitos alimentarios, sedentarismo) 1,3.

En los pacientes con trasplante renal, además de los factores que afectan a la población general, se asocian otros factores que influirían también en este aumento de peso, tales como la dieta y el tratamiento inmunosupresor. Con el trasplante mejora el estado anoréxico, hipercatabólico, y psicológico del paciente, lo que propicia un incremento en el apetito, el paciente pasa de una dieta extremadamente restringida a muy variada, sintiendo un mayor bienestar, resultando difícil controlar la alimentación. Algo parecido pasaría con el uso de esteroides sobre todo en los primeros meses del trasplante debidos a sus efectos metabólicos y al aumento del apetito del paciente ${ }^{1,3,5}$.

Por todo lo anterior y porque hemos observado que los pacientes que acudían a nuestra consulta ganaban peso durante el primer año, decidimos realizar este estudio con el fin de averiguar que estaba ocurriendo en nuestros trasplantados y adicionalmente detectar si habían aumentado los factores de riesgo, como la hipertensión, dislipemias diabetes, sobrepeso y obesidad, pudiendo afectar a la supervivencia del injerto.

\section{Objetivo}

Conocer la evolución del peso de nuestros pacientes durante el primer año del trasplante, y analizar su relación con las distintas variables antropométricas, demográficas y clínicas asociadas al paciente: edad, sexo, Índice Masa Corporal (IMC), tipo de tratamiento dialítico, enfermedad causante de la Insuficiencia Renal (IR), Índice de Filtrado Glomerular (IFG) y tratamiento inmunosupresor.

Determinar la asociación, si existe, entre la ganancia de peso y la aparición de factores de riesgo, como la hipertensión, diabetes y dislipemias.

\section{Material y método}

Estudio retrospectivo y analítico de los datos extraídos de la historia de los pacientes trasplantados en el Hospital Virgen de la Salud de Toledo. La muestra la formaron 100 pacientes con un año de evolución en el trasplante, de una población total de 159 trasplantados desde marzo 2007 hasta febrero de 2013, excluyéndose los 11 pacientes que no cumplieron el año de vida del injerto, lo que supone un $68 \%$, elegidos de forma aleatoria y representativa. Todos los injertos fueron de cadáver. Se elaboró una gráfica, para la recogida de datos antes del injerto, a los tres, seis y doce meses del trasplante. Datos como la edad, talla, peso, sexo, patología causante de la Enfermedad Renal, tipo del tratamiento dialítico, número de injertos en cada paciente, fecha del trasplante, tratamiento con inmunosupresores, antidiabéticos, hipotensores y antilipídicos, y datos bioquímicos y se calculo el índice de masa corporal, según la clasificación de la OMS (Tablal).

Tabla 1. Clasificación del sobrepeso y obesidad según el índice de masa corporal (IMC).

\begin{tabular}{|l|c|}
\hline \multicolumn{1}{c}{ IMC = Peso $(\mathrm{kg}) /$ Talla $^{2}\left(\mathrm{~kg} / \mathrm{m}^{2}\right)$} \\
\hline Bajopeso & $<18,5 \mathrm{~kg} / \mathrm{m}^{2}$ \\
\hline Normopeso & $18,5-24,9 \mathrm{~kg} / \mathrm{m}^{2}$ \\
\hline Sobrepeso grado I & $25-26,9 \mathrm{~kg} / \mathrm{m}^{2}$ \\
\hline Sobrepeso grado II (preobesidad) & $27-29,9 \mathrm{~kg} / \mathrm{m}^{2}$ \\
\hline Obesidad tipo I & $30-34,9 \mathrm{~kg} / \mathrm{m}^{2}$ \\
\hline Obesidad tipo II & $35-39,9 \mathrm{~kg} / \mathrm{m}^{2}$ \\
\hline Obesidad tipo III (mórbida) & $40-49,9 \mathrm{~kg} / \mathrm{m}^{2}$ \\
\hline Obesidad tipo IV (extrema) & $>50 \mathrm{~kg} / \mathrm{m}^{2}$ \\
\hline
\end{tabular}

La ganancia de peso total se calculó por la diferencia entre el peso a los doce meses y el peso inicial en el momento de trasplante, también se determinaron las ganancias parciales de peso $\mathrm{al}^{\circ}$ y $2^{\circ}$ y $4^{\circ}$ trimestre. 
Definimos ganancia de peso de un paciente, en $\mathrm{kg}$, cuando la diferencia entre el peso a los doce meses y el peso en el momento de trasplante es $>0 \mathrm{~kg}$ y no ganancia de peso en el paciente cuando la diferencia es $\leq 0 \mathrm{~kg}$.

Definimos como pacientes hipertensos, diabéticos, y dislipidemicos a los pacientes que tomaban uno o más medicamentos para el tratamiento de estas patologías.

El filtrado glomerular se estimó a partir de la creatinina sérica corregida con la superficie corporal, de cada paciente, mediante la fórmula de Cockcroft \& Gault.

Todos los datos de los pacientes fueron volcados en un Excel creando una base de datos de los pacientes para su tratamiento y análisis, el cálculo de parámetros estadísticos se realizó con la aplicación SPSS Statistics 22.

\section{Resultados}

De los 100 pacientes analizados, la Tabla 2 nos muestra datos socio demográfico, antropométrico, clínicos y bioquímico obtenidos.

Tabla 2. Datos de los pacientes trasplantados incluidos en la muestra.

\begin{tabular}{|c|c|c|c|}
\hline Variables & Conceptos & Datos & $\%$ \\
\hline Muestra & Trasplantados $\geq 1$ año & 100 & 100 \\
\hline \multirow[t]{2}{*}{ Sexo } & Hombres & 56 & 56 \\
\hline & Mujeres & 44 & 44 \\
\hline \multirow[t]{6}{*}{ Edad del paciente Fecha trasplante } & Menor de 30 años & 4 & 4 \\
\hline & Entre 30 y 39 años & 7 & 7 \\
\hline & Entre 40 y 49 años & 20 & 20 \\
\hline & Entre 50 y 59 años & 21 & 21 \\
\hline & Entre 60 y 69 años & 29 & 29 \\
\hline & Mayor de 70 años & 19 & 19 \\
\hline \multicolumn{2}{|l|}{ Edad media del paciente Fecha trasplante } & \multicolumn{2}{|c|}{$56,17 \pm 13,86$ años } \\
\hline \multirow[t]{6}{*}{ Etiología de la Nefropatía } & No filiada & 27 & 27 \\
\hline & Glomerular & 23 & 23 \\
\hline & Poliquistosis & 14 & 14 \\
\hline & Diabetes & 13 & 13 \\
\hline & Intesticial & 11 & 11 \\
\hline & Otras & 12 & 12 \\
\hline \multirow[t]{3}{*}{ Patología asociada Fecha trasplante } & Hipertensión & 88 & \\
\hline & Diabetes & 26 & \\
\hline & Dilipemias & 41 & \\
\hline \multirow[t]{2}{*}{ Tratamiento de la I.R.C. } & HD & 87 & 87 \\
\hline & DP & 13 & 13 \\
\hline \multirow[t]{2}{*}{ Número de trasplante } & $1^{\circ}$ & 100 & 100 \\
\hline & $2^{\circ}$ y $3^{\circ}$ & 10 & 10 \\
\hline \multicolumn{2}{|l|}{ Valor medio creatinina sérica } & \multicolumn{2}{|c|}{$1,36 \pm 0,35 \mathrm{mg} / \mathrm{dl}$} \\
\hline \multicolumn{2}{|l|}{ Rango creatinina } & \multicolumn{2}{|c|}{ 2,14 mínimo / 0,74 máximo 2,88 } \\
\hline \multicolumn{2}{|c|}{ Valor medio filtrado gromerular (Cok. Gault corregido) } & \multicolumn{2}{|c|}{$60,65 \pm 20,34 \mathrm{ml} /$ minuto } \\
\hline Procedencia injerto renal & Donante cadáver & 100 & 100 \\
\hline \multirow[t]{2}{*}{ Protocolo inmunosupresor } & EsteroidestTacrolimuss+Micofelonato & 95 & 95 \\
\hline & Otros & 5 & 5 \\
\hline
\end{tabular}

Predominan los hombres $56 \%$ frente a las mujeres $44 \%$, la edad promedio fue de $56,17 \pm 13,86$ años, el $48 \% \geq$ de 60 años, la etiología "no filiada" fue la que más se repitió con un $27 \%$, seguida de la glomerular con un $23 \%$, y el $95 \%$ recibió idéntico protocolo de inmunosupresión (Esteroides + Tacrolimus + Micofenolato).

El filtrado glomerular medio $(F G)$, medido por la fórmula de Cokcroft \& Gault fue del 66,35 $\pm 27,5$ $\mathrm{ml} /$ minuto. El $52 \%$ tenían al año valores de $\mathrm{FG}>$ $60 \mathrm{ml}$. /minuto. Un 4\% tenían hiperfiltración > 130 $\mathrm{ml} . / \mathrm{min}$., asociados a IMC $>30 \mathrm{~kg} / \mathrm{m}^{2}$. El valor medio de la creatinina sérica fue $1,36 \pm 0,35 \mathrm{mg}$. / dl., con un rango de la variable de 2,14 , mínimo 0,74 y máximo $2,88 \mathrm{mg} . / \mathrm{dl}$.

Al año de trasplante 93 pacientes (93\%) ganaron peso, el Gráfico 1 nos muestra la ganancia media de peso $7,21 \pm 5,84 \mathrm{~kg}, 22$ pacientes un $23,7 \%$ ganaron $\geq$ $10 \mathrm{~kg}$. El aumento de peso fue mayor en los primeros meses de trasplante.

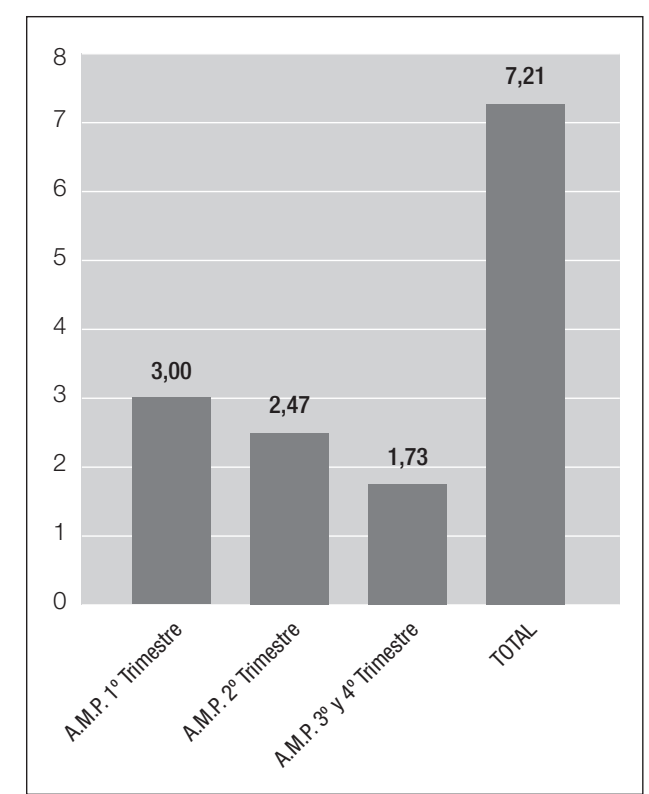

Gráfico 1. Evolución peso 93 pacientes año postrasplante aumento medio peso (A.M.P.) en Kg.

La Tabla 3 nos muestra la distribución de los pacientes de acuerdo al IMC, antes y al año del trasplante. El paciente de peso normal y los 15 pacientes de sobrepeso que pasaron a obesos tuvieron una ganancia media al año de $12,78 \mathrm{~kg}$. Los 17 pacientes de peso normal que pasaron a sobrepeso tuvieron una ganancia media al año de $9,39 \mathrm{~kg}$. 
Tabla 3. IMC de los pacientes pre trasplante y pos trasplante.

\begin{tabular}{|c|c|c|c|c|c|c|c|}
\hline IMC & $\begin{array}{l}\mathrm{N}^{0} \text { pac. } \\
\text { Pret. }\end{array}$ & $\begin{array}{l}\% \text { n }^{0} \text { pac. } \\
\text { Pret. }\end{array}$ & $\begin{array}{l}\mathrm{N}^{0} \text { pac. } \\
\text { Post. }\end{array}$ & $\begin{array}{c}\% \text { n }^{0} \text { pac. } \\
\text { Post. }\end{array}$ & AMP & $\Delta$ Obesos & $\underset{\text { sobrepeso }}{\Delta}$ \\
\hline BAJO PESO & 5 & $5 \%$ & 2 & $2 \%$ & 4,32 & & \\
\hline NORMAL & 35 & $38 \%$ & 20 & $22 \%$ & 6,91 & & \\
\hline SOBREPESO & 35 & $38 \%$ & 37 & $40 \%$ & 8,06 & -1 & -17 \\
\hline OBESIDAD & 18 & $19 \%$ & 34 & $37 \%$ & 6,95 & -15 & 17 \\
\hline TOTAL/MED. & 93 & $100 \%$ & 93 & $100 \%$ & 7,21 & 16 & \\
\hline \multicolumn{8}{|c|}{ OTROS INDICADORES } \\
\hline Peso medio & \multicolumn{2}{|c|}{$70,54 \pm 14,17 \mathrm{~kg}$} & \multicolumn{2}{|c|}{$77,76 \pm 16,25 \mathrm{~kg}$} & & & \\
\hline IMC medio & \multicolumn{2}{|c|}{$26,28 \pm 4,75 \mathrm{~kg} / \mathrm{m}^{2}$} & \multicolumn{2}{|c|}{$28,94 \pm 5,38 \mathrm{~kg} / \mathrm{m}^{2}$} & & & \\
\hline
\end{tabular}

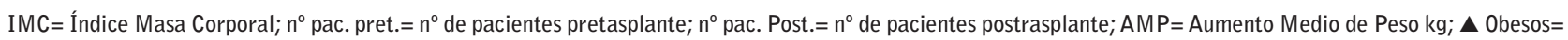
$\mathrm{n}^{\circ}$ de pacientes que pasan de otros IMC al IMC de obesos; $\boldsymbol{\Delta}$ Sobrepesos $=\mathrm{n}^{\circ}$ pacientes que pasan de otros IMC al IMC de sobrepeso.

Prevalencia sobrepeso y obesidad antes trasplante

Prevalencia sobrepeso y obesidad postrasplante

$\Delta$ Prevalencia sobrepeso y obesidad antes trasplante
$57 \% \quad$ Prevalencia obesidad antes trasplante

$76 \% \quad$ Prevalencia obesidad postrasplante

19\% $\Delta$ Prevalencia de obesidad postrasplante
$19 \%$

$37 \%$

$17 \%$
Los hombres ganaron 7,57 kg, y las mujeres $6,79 \mathrm{~kg}$. Por edades, la mayor ganancia de peso corresponde a los pacientes más jóvenes $\leq 49$ años con una media de $9,33 \mathrm{~kg}$. En los pacientes de Hemodiálisis la ganancia de peso fue de $7,36 \mathrm{~kg}$, mientras que los pacientes de Diálisis Peritoneal la ganancia fue de
$6,11 \mathrm{~kg}$ y el aumento de peso en pacientes de diálisis, fue mayor en el tercer y cuarto trimestre.

La Tabla 4, nos muestra el aumento de peso relacionado con la etiología de la Nefropatía.

Tabla 4. Aumento Medio de peso (AMP) dependiendo de la etiología de la Nefropatía.

\begin{tabular}{|l|c|c|c|c|c|c|c|}
$\begin{array}{c}\text { Etiología } \\
\text { Nefropatía }\end{array}$ & No Filiada AMP & $\begin{array}{c}\text { Glomerular } \\
\text { AMP. }\end{array}$ & $\begin{array}{c}\text { Poliquistosis } \\
\text { AMP. }\end{array}$ & $\begin{array}{c}\text { Diabetes } \\
\text { AMP. }\end{array}$ & $\begin{array}{c}\text { Intesticial } \\
\text { AMP. }\end{array}$ & Otras AMP. & Total AMP. \\
\hline$N^{0}$ pacientes & 25 & 23 & 11 & 12 & 11 & 11 & 93 \\
\hline Ganancia Peso & 8,65 & 6,30 & 5,86 & 7,28 & 7,06 & 7,28 & 7,21 \\
\hline
\end{tabular}

La Tabla 5, Evolución de los principales factores de riesgo. En la fase pretasplante 25 pacientes eran diabéticos, 20 con sobrepeso y obesidad, al año te- níamos 39, de los cuales 34 con sobrepeso y obesidad, el aumento de estos 14 pacientes, tiene su origen en 4 de sobrepeso y 7 de obesidad.

Tabla 5. Evolución de los principales factores de riesgo (Diabetes, Hipertensión y Dislipemias) pretrasplante y postrasplante.

\begin{tabular}{|c|c|c|c|c|c|c|c|}
\hline DIABETES & IMC pret. & IMC post. & DIAB. pret. & \% DIAB. pret. & DIAB. post. & \% DIAB. post. & $\triangle$ DIAB.AÑO \\
\hline Bajo Peso & 5 & 2 & 0 & $0 \%$ & 1 & $1,08 \%$ & $1,08 \%$ \\
\hline Normal & 35 & 20 & 5 & $5,38 \%$ & 4 & $4,30 \%$ & $-1,08 \%$ \\
\hline Sobrepeso & 35 & 37 & 10 & $10,75 \%$ & 13 & $13,98 \%$ & $3,23 \%$ \\
\hline Obesidad & 18 & 34 & 10 & $10,75 \%$ & 21 & $22,58 \%$ & $11,83 \%$ \\
\hline Total & 93 & 93 & 25 & $26,88 \%$ & 39 & $41,94 \%$ & $15,05 \%$ \\
\hline HTA & IMC Pret. & IMC Post. & HTA. pret. & $\%$ HTA. pret. & HTA. post. & \% HTA. post. & $\triangle$ HTA. AÑO \\
\hline Bajo Peso & 5 & 2 & 4 & $4,30 \%$ & 2 & $2,15 \%$ & $-2,15 \%$ \\
\hline Normal & 35 & 20 & 29 & $31,18 \%$ & 19 & $20,43 \%$ & $-10,75 \%$ \\
\hline Sobrepeso & 35 & 37 & 32 & $34,41 \%$ & 34 & $36,56 \%$ & $2,15 \%$ \\
\hline Obesidad & 18 & 34 & 16 & $17,20 \%$ & 31 & $33,33 \%$ & $16,13 \%$ \\
\hline Total & 93 & 93 & 81 & $87,10 \%$ & 86 & $92,47 \%$ & $5,38 \%$ \\
\hline DILIPEMIAS & IMC Pret. & IMC Post. & DISLIP. Pret. & \% DISLIP. Pret. & DISLIP. Post. & \% DISLIP. Post. & $\triangle$ DISLIP. AÑO \\
\hline Bajo Peso & 5 & 2 & 1 & $1,08 \%$ & 1 & $1,08 \%$ & $0,00 \%$ \\
\hline Normal & 35 & 20 & 9 & $9,68 \%$ & 10 & $10,75 \%$ & $1,08 \%$ \\
\hline Sobrepeso & 35 & 37 & 18 & $19,35 \%$ & 32 & $34,41 \%$ & $15,05 \%$ \\
\hline Obesidad & 18 & 34 & 10 & $10,75 \%$ & 26 & $27,96 \%$ & $17,20 \%$ \\
\hline Total & 93 & 93 & 38 & $40,86 \%$ & 69 & $74,19 \%$ & $33,33 \%$ \\
\hline
\end{tabular}

DIAB= DIABÉTICOS; HTA= HIPERTENSOS; DISLIP= DISLIPÍDICOS; pret= pretasplante; Post= postrasplante s= Incremento de DIAB; HTA; DISLIPÍDICOS 
Casi la totalidad de nuestros pacientes, 81 eran hipertensos de los cuales 48 con sobrepeso y obesidad, al año la cifra se aumenta a 86 hipertensos, 65 con sobrepeso y obesidad, el aumento de hipertensos 5 pacientes, tiene su origen 2 de sobrepeso y 2 en obesidad.

En la etapa pretasplante 38 pacientes dislipidémicos, de los cuales 28 con sobrepeso y obesidad, aumentando al año a 69 pacientes, 32 con sobrepeso y 26 con obesidad. riesgo en obesos.

Tabla 6. Evolución de los principales factores de riesgo asociados Hipertensión, Diabetes y Dislipemias en pacientes obesos.

\begin{tabular}{c|c|c|c|}
\hline TRASPLANTADOS & OBESOS & $\begin{array}{c}\text { HIPERTENSOS + } \\
\text { DIABÉTICOS + } \\
\text { DISLIPÍDIC OS }\end{array}$ & $\%$ \\
\hline Pretrasplante & 18 & 5 & $5 \%$ \\
\hline Postrasplante & 34 & 15 & $16 \%$ \\
\hline
\end{tabular}

La tabla 6, nos muestra la asociación de factores de

\section{Discusión}

El trasplante renal es el mejor Tratamiento Sustitutivo de la ERC con él se ha logrado una supervivencia del injerto del $90 \%$ al año del trasplante ${ }^{22}$. Con la aparición de nuevos inmunosupresores en la década de los 90, la supervivencia del injerto mejoro a corto plazo, pero a largo plazo los cambios han sido mínimos ${ }^{1,3,6}$. Las estrategias actuales van dirigidas a prolongar la supervivencia del trasplante.

La causa principal de la perdida de la función renal es la nefropatía crónica del injerto, motivada por un proceso multifactorial, inmunológico y no inmunológico que genera un deterioro progresivo de la función renal. También están implicados la nefrotoxicidad de los inmunosupresores, la hipertensión arterial, la dislipemias y la diabetes ${ }^{1,7}$.

La ganancia de peso es un hecho frecuente en el trasplante renal. La

Un $13 \%$ de los pacientes antes del trasplante tenían asociados los tres factores e riesgo del Síndrome metabólico, (DM, HTA, DLPT) aumentando el 30\% al año del trasplante.

El Gráfico 2 nos muestra la correlación existente entre el aumento de pacientes con sobrepeso y obesos y el aumento de los factores de riesgo, diabetes y dislipemias al año del trasplante. Un aumento del $19 \%$ en pacientes con sobrepeso y obesos tiene un incremento del $15 \%$ en diabéticos y un $32 \%$ en dilipidemicos.

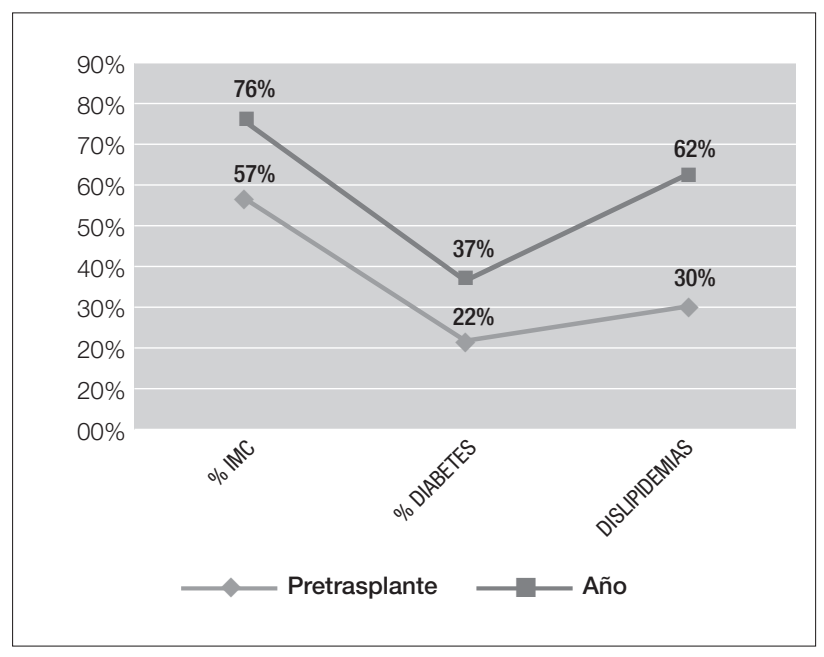

Gráfico 2. IMC (sobrepeso y obesidad) vinculado a factores de riesgo. prevalencia de obesidad antes del trasplante, oscila entre el $10 \%{ }^{8,9}$ y el $19 \%,{ }^{10,11}$ similar a la población general, afectando en el postrasplante al $36 \%$ de los pacientes ${ }^{11}$.

Merion \& col. constato una ganancia de peso de $9 \mathrm{~kg}$ en pacientes no obesos, y de $14 \mathrm{~kg}$ en los obesos ${ }^{12}$, mientras que Gill \& col. reporto un aumento de peso de $8,5 \mathrm{~kg}$ en ambos grupos de pacientes ${ }^{13}$, Clunk y col. analizo los factores asociados a la ganancia de peso post trasplante y constato que la raza negra el sexo femenino y una edad relativamente joven $(10,3$ $\mathrm{kg}$ ) se asociaban a una mayor ganancia de peso, otros trabajos también lo demuestran, aunque existen estudios contradictorios en cuanto al sexo $0^{14}$.

La mayoría de los estudios han demostrado que la obesidad se asocia a una menor supervivencia del injerto y del paciente incluso con el riñón funcionarte ${ }^{1}$.

Nuestro estudio ha podido constatar muchas de estas afirmaciones. La supervivencia del injerto al año es del $93 \%$. A corto plazo no se ha demostrado alteración de la función renal relacionada con el aumento de peso, siendo necesario un estudio a medio y largo plazo, para poder demostrar la probable afectación en la supervivencia del injerto.

La ganancia de peso media al año del trasplante, fue de $7,21 \pm 5,84 \mathrm{~kg}$, ligeramente inferior a otras series $^{3,12}$, así como la ganancia excesiva de peso $23,7 \%$ ganaron $\geq 10 \mathrm{~kg}$, la mayor ganancia fue en el primer 
y segundo trimestre, relacionado probablemente con el tratamiento inmunosupresor.

Al contrario que otros estudios 1,3 las mujeres ganaron menos peso, poco significativo, los pacientes jóvenes ganaron más peso, al igual que otras series, casuística idéntica a la población en general.

En nuestra serie la enfermedad causante de la insuficiencia renal con mayor ganancia de peso es de etiología no filiada, este dato carece de valor estadístico.

No podemos referenciar ganancias de pesos por el tratamiento inmunosupresor porque al $95 \%$ se trató con el mismo protocolo.

Al igual que en otros estudios la prevalencia de diabetes y dislipemias fue mayor en pacientes con sobrepeso y obesidad ${ }^{1,3}$.

La hipertensión arterial fue otro factor de riesgo analizado, el estudio nos muestra una prevalencia casi total en la población antes y después del trasplante, con independencia de la ganancia de peso. Hecho que corroboran otros estudios ${ }^{3,15}$.

No se puede asegurar que todos los resultados obtenidos sean debidos a la sobrecarga de peso ya que es una patología multifactorial, pero pueden servir para el diseño e implementación de modificaciones sobre todo en los estilos de vida y alimentarios del paciente, antes y después del trasplante, que es donde la enfermería puede actuar con la educación del paciente.

\section{Conclusiones}

La mayoría de los pacientes aumentaron significativamente de peso al año del trasplante, con la mayor incidencia en el primer y segundo trimestre, muchos de los trasplantados ingresan en las categorías de IMC de sobrepeso y obesidad, asociándose con varios de los factores de riesgo del síndrome metabólico, hipertensión arterial, dislipemias y diabetes.

No parece que la ganancia de peso, acorto plazo influya sobre la función del injerto.

La enfermería tendría un papel importante actuando precozmente, antes y después del trasplante, en la educación del paciente, fomentando hábitos de vida saludable, dieta y actividad física adecuada, control de la presión arterial y azúcar en sangre. Motivar a mantener un peso normal, puede reducir el riesgo de enfermedad renal y prevenir o disminuir las complicaciones asociadas al sobrepeso y obesidad.
Recibido: 9 Septiembre 2014

Revisado: 12 Septiembre 2014

Modificado: 12 Septiembre 2014

Aceptado: 12 Septiembre 2014

\section{Bibliografía}

1. Nuria Esforzado, Federico Cofan, Nahyr Schinca, Sonia Hervas, Monserrat Pujades. Obesidad en el trasplante renal. Actividad Dietética. N 33. 2007.

2. Marcén $R$, Fernández $A$, Pascual $J$, Teruel JL, Villafruela JJ, Rodríguez Net al. High body mass index and posttransplant weight gain are not riskfactors for kidney graft and patientoutcome. Transplant Proc 2007;39:2205-7.

3. Gerardo Boroto Díaz, Mardia Quintanilla Andino, Maricela Barceló Acosta, Lisandra Cabrera Valdés. Ganancia de peso, dismetabolia y función renal al año del trasplante renal. Revision Cubana Aliment. Nutri. 2012; 22(2): 186-202.

4. Reaven G. The metabolic syndrome or the insulin resistance syndrome? Different names, different concepts and different goals. Endocrino Metab Clin North Am 2004;33:283-303.

5. Gerardo Boroto Díaz, Maricela Barceló Acosta, Carlos Guerrero, Díaz, Jesús Barreto Penie. Estado nutricional del paciente trasplantado renal. Asociación con la función del injerto renal, la insulinoresistencia y las complicaciones metabólicas. Revi C8uvana Nutr 2010;20(2):213225.

6. El-Zoghby ZM. Identifying specificcauses of kidney allograft loss. Am JTransplant 2009;9:527.

7. Kasiske BL, Israni AK, Snyder P,Melissa A. The relationship betweenkidney function and long-term graftsurvival after kidney transplant. Am JKidney Dis 2011;57:466-75.

8. Pirsch, JD.; Armbrust, MJ.; Knechtle, SJ.; D’Alessandro, AM.; Sollinger, HW.; Heisey, DM.; Belzer, F0. Obesitya risk factor following renal transplantation. Transplantation 1995,59:631633. 
9. Howard, RJ.; Thai, VB.; Patton, PR.;Hemming, AW.; Reed, AI.; Van der Werf, WJ.; Fujita, S.; Karlix, JL.;Scornik, JC. Obese Kidney Transplant Recipients have good outcomes. Transplant Proc 2001, 33:3420-3421.

10. Pfeiffer, T.; Albrecht, KH.; Wagner, K.; Herget, S.; Eigler, FW. The effectof obesity on outcome of kidneytransplantation. Med Klin 1994,89:635639.

11. Cofán $F$, Vela $E$, Clèries $M$; for the Catalan Renal Registry.Obesity in renaltransplantation: Analysis of 2691 patients. Transp Proc 2005;37:3695-7.

12. Merion, RM.;Twork,AM.; Rosenberg,L.; Ham,JM.; Burtch, GD.; Turcotte,JG.; Rocher, LL.; Campbell, DA. Jr. Obesity and renal transplantation. Surg Gynecol Obstet 1991, 172:367-376.
13. Gill, IS.; Hodge, EE.; Novick, AC.; Steinmuller, DR.; Garred, D. Impact of obesity on renal transplantation. Transplant Proc 1993, 25:10471048.

14. Clunk, JM.; Lin, Ch.; Curtis, JJ.Variables affecting weight gain in renal transplant recipients. Am J Kidney Dis 2001, 38(2):349-353.

15. M. Rengel. Obesidad e hipertensión arterial en pacientes con trasplante renal. Nefrología. Vol. XXII Suplemento 2. 2002. 